\title{
Management Strategies of Patients with Neuromyelitis Optica Spectrum Disorder During the COVID-19 Pandemic Era
}

This article was published in the following Dove Press journal:

Therapeutics and Clinical Risk Management

\author{
Sherif M Hamdy \\ Maged Abdel-Naseer (D) \\ Hatem S Shehata (D) \\ Nevin M Shalaby \\ Amr Hassan (D) \\ Alaa Elmazny (D) \\ Ehab Shaker \\ Mona AF Nada (D) \\ Sandra M Ahmed \\ Mohamed I Hegazy \\ Husam S Mourad (D) \\ Ahmed Abdelalim (1D) \\ Rehab Magdy \\ Alshimaa S Othman (ID \\ Doaa A Mekkawy \\ Nirmeen A Kishk (D) \\ Neurology Department, Faculty of \\ Medicine, Cairo University, Cairo, Egypt
}

Correspondence: Alaa Elmazny 66-Manial-Street, Cairo, Egypt

Tel +9665360I2237

Email alaa_elmazny@kasralainy.edu.eg

\begin{abstract}
The ongoing coronavirus (COVID-19) pandemic is a global health emergency of international concern and has affected management plans of many autoimmune disorders. Immunosuppressive and immunomodulatory therapies are pivotal in the management of neuromyelitis optica spectrum disorder (NMOSD), potentially placing patients at an increased risk of contracting infections such as COVID-19. The optimal management strategy of NMOSD during the COVID-19 era remains unclear. Here, however, we examined the evidence of NMOSD disease-modifying therapies (DMTs) use during the present period and highlighted different scenarios including treatment of relapses as well as initiation and maintenance of DMTs in order to optimize care of NMOSD patients in the COVID-19 era. Keywords: neuromyelitis optica spectrum disorder, COVID-19, pandemic
\end{abstract}

\section{Introduction}

Severe acute respiratory syndrome coronavirus 2 (SARS-CoV-2), identified as the cause of coronavirus disease 2019 (COVID-19), is the third known virus of the coronaviridae family to cause outbreaks of severe acute respiratory syndrome. ${ }^{1}$

Since the recognition of the disease as a pandemic, concerns have arisen over patients suffering immune-mediated illnesses requiring management with immunomodulatory (IM) or immunosuppressant (IS) therapies, given their higher risk for suffering more severe forms of COVID-19. ${ }^{2}$

This review discusses the challenges in treating neuromyelitis optica spectrum disorder (NMOSD) in the COVID-19 era and proposes algorithms for tailoring potential management of acute relapses as well as the use of disease-modifying therapies (DMTs) during this critical period.

\section{Pathophysiology of Neuromyelitis Optica Spectrum Disorder (NMOSD)}

The NMOSD represents a group of immunologically mediated, inflammatory central nervous system (CNS) disorders, involving the production of auto-reactive antibodies (Abs) against various neuroglial structures. Autoantibodies against water channel aquaporin-4 (Anti AQP-4 IgG1) are the most common Abs, ${ }^{3}$ although other known overlapping Abs include those against myelin oligodendrocyte glycoprotein IgG (anti-MOG) ${ }^{4}$ and glial fibrillary acidic protein (GFAP). ${ }^{5}$ Cell-mediated immunity (CMI) contributes to the pathophysiology, where polarization of T-lymphocytes 
towards helper Th-17 and Th-2 subtypes results in cytokine network alteration ${ }^{6,7}$ and promotes pro-inflammatory cascades and milieu conducive for B-lymphocytes. A major pathophysiologic role is played by Interleukin (IL) 6, a cytokine specifically involved in relapse severity, CNS injury and degree of disability. ${ }^{8}$ Disability in NMOSD is highly related to relapses; reduction of relapse frequency and adequate relapse management are thus of paramount importance. ${ }^{9}$

\section{Pathophysiology of COVID-I 9}

Innate immunity is initially activated following SARSCoV-2 infection, with increased expression of inflammatory markers (eg CRP, procalcitonin, ferritin) and type-1 interferons (IFN) in an attempt to limit viral replication and spread. Ensuing activation of phagocytosis targets infected cells. $^{2}$

Eventually, adaptive immunity is activated, and a major role is played by CMI via activation of virus-specific T-cytotoxic cells (Tc-CD8). Antibody-mediated immunity and the complement cascade (particularly involving C3, C5) additionally aid antibody-dependent cellular cytotoxicity. ${ }^{10}$

Under optimal conditions, the immune response is well regulated and results in effective viral clearance and a good clinical outcome. ${ }^{2}$ However, in other conditions, viral virulence mechanisms result in dysregulation of the immune response and unrestrained viral replication, increased viral load, delayed IFN production, accumulation of neutrophils and monocytes, and reduction in B and T cells. A storm of pro-inflammatory cytokines follows, characterized by pooling of IL-2, $-6,-8,-10$, and -21 , and tumour necrosis factor (TNF)- $\beta .{ }^{11}$ This creates a vicious circle of overactive innate and dampened adaptive immune responses that are usually associated with clinical deterioration, acute lung injury, acute respiratory distress syndrome, thrombophilia and multiple systems organ failure. ${ }^{4}$

Older age, male gender, smoking, the presence of comorbidities (particularly diabetes, cardiac and respiratory diseases) and immunosuppression were all found to be factors predisposing to dysregulation of the immune response and thus a worse clinical course among COVID19 positive patients. ${ }^{2,4,12}$

\section{Dilemmas in NMOSD Management During COVID-I 9}

The ongoing COVID-19 pandemic has created unparalleled challenges for neurologists to deliver the best care to patients suffering NMOSD. Questions that are yet to be addressed include: (1) whether exposure to certain DMT regimens poses a higher risk of severe COVID-19 infection that may require discontinuation, transient interruption or substitution of therapies, encouraging adoption of appropriate risk-stratification strategies in the context of individual patient profiles as well as of drug properties; (2) whether infection with COVID-19, even if asymptomatic, can precipitate acute relapses in otherwise stable patients, as can any other infection although shared immunopathological pathways between the two disorders would necessitate further risk stratification.

Limiting unnecessary procedures in medical centers to preserve resources and reduce person-to-person contact warrant consideration (eg delaying follow-up MRI scans and reducing the frequency of routine laboratory monitoring in stable patients). Further measures, such as suspending non-essential in-person consultations and clinic visits or substituting it with remote interventions should also be encouraged (eg checking laboratory or MRI results through telecommunications). ${ }^{13}$

Tailored recommendations for neurologists managing NMOSD patients are currently needed, especially in the case of patients acquiring COVID-19.

\section{Relapse Management}

In patients with NMOSD, studies have shown that permanent damage from relapses leads to cumulative disability; therefore, the consensus among NMOSD experts is that every relapse requires treatment and that rescue medications should be initiated as early as possible. Preventive treatments should be continued during relapse management as they play a role in recovery and ameliorating relapse severity. ${ }^{5,14,15}$

Treatment decisions regarding NMOSD relapses should be individualized according to COVID-19 suspicion, relapse manifestations, need for hospitalization and previous patient responses. Escalation of relapse treatment improves outcome, and the sequence of therapies is crucial until a satisfactory response is achieved. Administration of intravenous methylprednisolone, $1 \mathrm{~g}$ daily for 5-7 consecutive days, is typically considered to be the initial treatment of choice and patients are encouraged to receive infusions at home whenever possible. ${ }^{16-19}$ However, in severe relapses (expanded disability status scale (EDSS) scores $\geq 2$ points), this first-line treatment is not sufficient and additional strategies are required to minimize any residual impairments. ${ }^{20-22}$ In view of the pathophysiology of NMOSD where a strong 
humoral response underlies the disease process, plasma exchange (PLEX) seems to be the most appropriate therapy in severe relapses and it is accepted as the second-line therapy for severe steroid-refractory NMOSD attacks with more favorable outcome. ${ }^{23,24}$ The American Society for Apheresis (ASFA) considers NMOSD to be a category II indication for PLEX.

With the evidence of complex interactions between the cytokine storm, inflammation, endothelial dysfunction, and thrombophilia resulting from COVID-19 infection ${ }^{25,26}$ PLEX offers effective removal of inflammatory cytokines from the blood, stabilization of endothelial membranes, and reversal of hypercoagulability. ${ }^{25-27}$

However, PLEX is an invasive procedure with a longer hospital stay as well as a higher risk of infections and hemodynamic instability when compared to intravenous immunoglobulin (IVIG), especially for patients on immunosuppressants. ${ }^{28}$ Hence, IVIG administration could be a relatively safer option with favorable results in relapse treatment in patients with neurological manifestations not warranting hospital admission ${ }^{29-31}$ (Figure 1).

Furthermore, high IVIG doses administered to patients with severe COVID-19 were found to successfully halt the progression of the illness and improve clinical outcomes. ${ }^{33,34}$

Monthly IVIG administration is also considered to be an effective treatment option in preventing NMOSD relapses in patients with anti-MOG disease, especially throughout the COVID-19 pandemic, owing to its immune-boosting and antiviral properties. ${ }^{35-37}$

\section{Initiation and Maintenance of DMTs}

Various immunosuppressive agents including azathioprine (AZA), mycophenolate mofetil (MMF), and cyclophosphamide (CTX), as well as monoclonal antibodies including rituximab (RTX), eculizumab, and tocilizumab have been reported effective in reducing the annualized relapse rate and stabilizing EDSS scores of NMOSD patients. ${ }^{38-42}$ The suggested treatment algorithm for the initiation and maintenance of DMTs is outlined in Figure 2.

\section{Azathioprine}

This purine antimetabolite is structurally similar to endogenous purine bases and interferes with DNA synthesis. This results in $\mathrm{T}$ cell apoptosis and subsequent immunosuppression. ${ }^{43}$ As an immunosuppressive medication, this drug increases the risk of bacterial, viral and fungal infection especially when combined with corticosteroids. ${ }^{43}$ Lymphopenia (especially grade 3 and $4 ;<500$ cells $/ \mathrm{mL}$ ) was found to increase the risk of opportunistic infection, so frequent WBC monitoring as well as dose reduction or even medication cessation should be considered. ${ }^{44,45}$

In the management of NMOSD, AZA is considered to be a first-line therapy, and no evidence to date has

New or worsening of neurological manifestation lasting $>24 \mathrm{hs}$

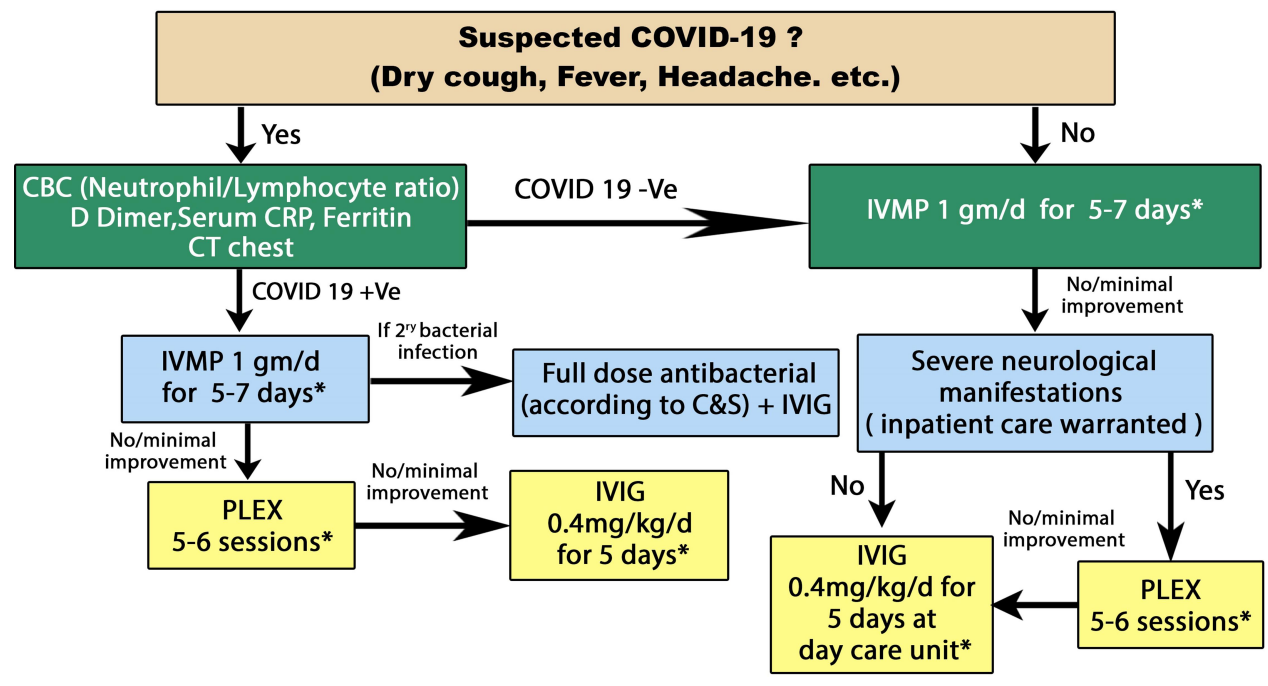

Figure I Management of a relapse in NMOSD patients during COVID-19 pandemic. ${ }^{32}$

*After improvement continue on oral steroids for 3-6 months and consider initiation of disease-modifying therapies in newly diagnosed patients.

Abbreviations: CBC, complete blood count; COVID-19, coronavirus disease 2019; CRP, C reactive protein; CT, computed tomography; IVIG, intravenous immunoglobulins; IVMP, intravenous methylprednisolone; NMOSD, neuromyelitis optica spectrum disorder; PLEX, plasma exchange. 


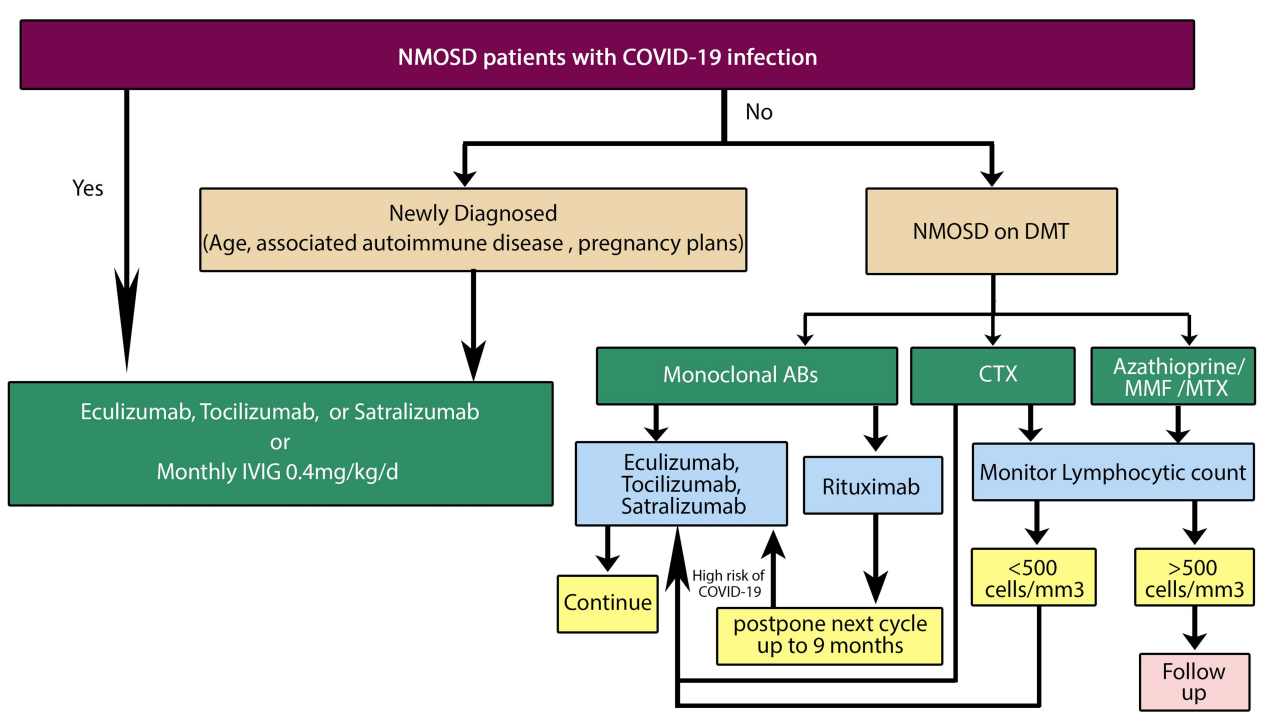

Figure 2 Algorithm for initiation and maintenance of DMTs in NMOSD patients during the COVID-19 pandemic.

Abbreviations: ABs, antibodies; COVID-19, coronavirus disease 2019; CTX, cyclophosphamide; DMT, disease modifying therapy; IVIG, intravenous immunoglobulins; MMF, mycophenolate mofetil; MTX, methotrexate; NMOSD, neuromyelitis optica spectrum disorder.

suggested that interrupting AZA treatment decreases risks posed by COVID-19. ${ }^{46}$

\section{Recommendations}

AZA can be continued as a maintenance therapy in NMOSD patients throughout the COVID-19 pandemic with regular blood count monitoring. Dosages can be adjusted based on individual patient profile, disease severity and lymphocytic counts. ${ }^{45}$ However, it is likely safer not to start AZA in newly diagnosed patients.

\section{Rituximab}

This monoclonal antibody against CD20-expressing B lymphocytes has not been found to increase the risk of viral infection; there is likewise no evidence to date that RTX increases the risk of developing COVID- $19 .{ }^{47}$ AntiCD20 therapies leave the CD8-positive T cell compartment of the immune system relatively intact, which is important to eradicate the virus. However, it is not preferable to initiate B cell-depleting agents during a pandemic because they may preclude the formation of long-lasting immunity against reinfection as well as response to vaccines when they become available. ${ }^{48}$

The timing of RTX doses can be determined by monitoring CD19+ CD27+ memory B cells and does not necessarily follow the 6-month interval dosing. Disease control in NMOSD patients can be achieved with RTX for an average of 6-9 months, ${ }^{49-51}$ and it is now obvious from the Swedish experience in multiple sclerosis that rituximab remains effective at controlling disease activity beyond 6 months. ${ }^{50}$

\section{Recommendations}

Initiation of RTX in NMOSD patients throughout the COVID-19 pandemic is not preferable. Stable patients already undergoing RTX treatment are advised to have their next infusion postponed for 3 months especially if CD19+ and CD27+ memory B cell counts are severely decreased.

In situations where CD19+ and CD27+ memory B cell levels have stabilized or when 9 months have passed since the prior infusion, the next medication dose cannot be postponed and it is recommended to either shift from RTX to one of the other approved safer monoclonal antibodies, or to continue the patient on the same drug after explaining potential risks and benefits.

\section{Mycophenolate Mofetil}

A prodrug of mycophenolic acid, MMF causes depletion of guanosine and deoxyguanosine in both $\mathrm{T}$ and B lymphocyte lines via inhibition of inosine-5'monophosphate dehydrogenase (type II), an enzyme that facilitates the production of an intermediate metabolite of guanosine. ${ }^{52}$ Net effects include the inhibition of de novo purine synthesis and the suppression of DNA synthesis with subsequent interference in both $\mathrm{T}$ and $\mathrm{B}$ lymphocyte proliferation, decrease in cytotoxic $\mathrm{T}$ cell activity and diminished antibody formation. ${ }^{53-55}$ Promising results 
have been reported in the setting of many immunemediated neurological illnesses including NMOSD. ${ }^{56,57}$

Compared to AZA, MMF was more effective at achieving remission in some patients with NMOSD and did so with fewer adverse events. ${ }^{58}$

This drug interferes with IL-17 production by Th17 cells. A potent pro-inflammatory cytokine that plays a crucial rule in COVID-19 infection, chronic use of MMF could have a protective role against serious clinical complications of the disease. ${ }^{59,60}$

\section{Recommendations}

It is likely safer not to start MMF in newly diagnosed NMOSD patients throughout the pandemic. For patients who are already being treated with MMF, treatment can be maintained with regular blood count monitoring.

\section{Methotrexate}

A folate antagonist that has multiple dose-related mechanisms of action, MTX inhibits dihydrofolate reductase and thus DNA synthesis in high-dose regimens and aminoimidazole-4-carboxamide ribonucleotide in low-dose regimens, ultimately interfering with T-cell function, augmenting the release of intra- and extracellular adenosine that suppresses inflammation, suppressing the release of pro-inflammatory cytokines (eg TNF- $\alpha$, IL-6, IL-8, IL-12) and enhancing the production of anti-inflammatory mediators (eg IL-10 and IL-1). ${ }^{61}$

In the treatment of NMOSD, MTX is considered to be a second-line option when first-line medications fail or are inappropriate. $^{62}$

In addition, MTX therapy is associated with increased risk of infection and myelosuppression ${ }^{63}$ while in up to $14 \%$ of patients treated with low-dose MTX, a troublesome adverse event mimicking symptoms of COVID-19, termed MTX-induced pneumonitis (MIP), has been reported. It typically presents with dyspnea, dry cough and fever, as well as ground glass opacities on chest CT imaging. Cessation of MTX and glucocorticoids typically results in a favorable outcome, although, in some cases, it may be fatal. ${ }^{64}$

An interim Australia/New Zealand recommendation for patients with severe inflammatory skin disorders was to reduce MTX dosing to less than $10 \mathrm{mg}$ /week to protect against COVID-19 infection. $^{45}$

\section{Recommendations}

As a second-line agent, it is preferable not to initiate MTX in NMOSD patients throughout the COVID-19 pandemic. Frequent clinical evaluations and complete blood count monitoring of patients treated with MTX are recommended; reduction of doses to less than $10 \mathrm{mg} /$ week should be considered for patients already undergoing MTX therapy based on individual profiles and disease severity. If patients develop MIP, are infected with COVID-19 or if lymphocytic count falls below 1100/mL, MTX should be stopped.

\section{Cyclophosphamide}

An alkylating antineoplastic agent, CTX exerts its effects via the active metabolite phosphoramide, resulting in crosslinkages within and between adjacent DNA strands, ultimately leading to induction of apoptosis. ${ }^{65}$ This medication also selectively suppresses $T$ cells and increases the secretion of Th2 cytokines (eg IL-4, IL-10) and decreases the secretion of INF-gamma and IL-12 both in the CSF and peripherally. ${ }^{66}$ Common adverse events include hemorrhagic cystitis, gonadal toxicity and myelosuppression ${ }^{67}$. Myelosuppression increases the risk of infection, which may trigger sepsis, particularly at high treatment doses ${ }^{68}$. Furthermore, immunosuppression induced by CTX in a murine model infected with influenza $A$ revealed viral persistence with prolonged shedding. ${ }^{69}$ The European Medicines Agency approves CTX as a second-line maintenance therapy for NMOSD patients, especially for those with comorbid systemic lupus erythematosus or systemic sclerosis.

\section{Recommendations}

It is not preferable to initiate CTX in NMOSD patients throughout the COVID-19 pandemic. As CTX is a secondline agent, the choice of another alternative as maintenance therapy is preferable.

\section{Eculizumab}

Eculizumab is a humanized monoclonal antibody approved for the treatment of NMOSD in patients who are AQP4-IgG-seropositive. It binds with high affinity to C5, thereby blocking the activation of the terminal complement pathway. ${ }^{70}$

The complements $\mathrm{C} 3 \mathrm{a}$ and $\mathrm{C} 5 \mathrm{a}$ in particular have very potent pro-inflammatory properties as they trigger neutrophil recruitment and activation, thus playing a vital role in the immune response to COVID-19 infection. Interestingly, anti-C5aR antibody was reported to alleviate lung and tissue damage in Middle East respiratory syndrome-related coronavirus (MERS-CoV)-infected mice. ${ }^{71}$

Despite the fact that experience with the novel monoclonal antibodies in NMOSD treatment is very limited, 
eculizumab, as a modulator of distal complement, was found to hold promise in the treatment of patients infected with COVID-19. ${ }^{72}$

Commonest reported adverse effects of eculizumab are headache $(27.0 \%)$, upper respiratory tract infection (25.5\%), nasopharyngitis (22.6\%) and bacterial pneumonia $(2.9 \%) .{ }^{73}$ Such adverse respiratory effects may either falsely suggest COVID-19 infection, or mask it.

\section{Recommendations}

Initiation and maintenance therapy with eculizumab in NMOSD patients throughout the COVID-19 pandemic is likely safe, even in patients with suspected or confirmed COVID-19. However, careful monitoring for possible upper respiratory infections as well as bacterial pneumonia is warranted.

\section{Tocilizumab}

Tocilizumab is a humanized monoclonal antibody that binds to IL-6 receptors and thus blocks their binding IL6, a pro-inflammatory cytokine with a major role in NMOSD pathogenesis. ${ }^{38}$ Tocilizumab is highly effective for aggressive cases of NMOSD refractory to RTX therapy. Notably, tocilizumab has an additional role in the effective management of chronic neuropathic pain and fatigue in NMOSD patients. ${ }^{74}$

In patients with COVID-19 infection, IL-6 levels are significantly elevated and associated with adverse outcomes. Thus, tocilizumab was found to effectively dampen the inflammatory response and improve patient outcomes. ${ }^{75-77}$

Tocilizumab is generally a safe drug with injection site reactions the commonest adverse effect (10\%). However, neutropenia, thrombocytopenia and elevated liver enzymes may rarely occur. ${ }^{78}$

Importantly, impaired IL-6 signaling results in normalization of CRP levels and body temperature even in the presence of systemic infection. ${ }^{79}$ Suspicion of COVID-19 infection in patients receiving IL-6 inhibitors should be high in the setting of relevant symptomology.

\section{Recommendations}

Initiation and maintenance therapy with tocilizumab in NMOSD patients throughout the COVID-19 pandemic is likely safe, even in patients with suspected or confirmed COVID-19 as tocilizumab is possibly dually effective.

\section{Satralizumab}

Satralizumab is another humanized monoclonal antibody that binds the IL- 6 receptor but exhibits a more favorable pharmacokinetic profile than tocilizumab due to a prolonged elimination half-life. ${ }^{80}$

As an IL-6 inhibitor, satralizumab is likely beneficial in the setting of COVID-19 infection; however, this drug has not been investigated in COVID-19 patients.

\section{Recommendations}

Initiation and maintenance therapy with satralizumab in NMOSD patients throughout the COVID-19 pandemic is likely safe even in patients with suspected or confirmed COVID-19 infection.

\section{Conclusions}

There is little evidence to formulate specific recommendations for patients with NMOSD receiving immunomodulatory treatment with regards to COVID-19. However, in everyday clinical practice throughout the pandemic, health care providers face several obstacles regarding acute and preventive management of NMOSD patients. Here, we listed recommendations based on the mechanism of action of each drug and its preferential effects on the immune system, taking into consideration currently available data, ongoing studies and expert opinion. However, treatment decisions regarding NMOSD patients should be individualized according to disease duration, course and severity, as well as COVID-19 risk.

\section{Disclosure}

The authors report no conflicts of interest in this work.

\section{References}

1. Zhu N, Zhang D, Wang W, et al. A Novel Coronavirus from Patients with Pneumonia in China, 2019. N Engl J Med. 2020;382(8):727-733. doi:10.1056/NEJMoa2001017

2. Yi Y, Lagniton PNP, Ye S, Li E, Xu R-H. COVID-19: what has been learned and to be learned about the novel coronavirus disease. Int J Biol Sci. 2020;16(10):1753. doi:10.7150/ijbs.45134

3. Wingerchuk DM, Banwell B, Bennett JL, et al. International consensus diagnostic criteria for neuromyelitis optica spectrum disorders. Neurology. 2015;85(2):177-189. doi:10.1212/WNL.0000000000001729

4. Chen G, Wu D, Guo W, et al. Clinical and immunological features of severe and moderate coronavirus disease 2019. J Clin Invest. 2020;130 (5):5. doi:10.1172/JCI137244

5. Kimbrough DJ, Fujihara K, Jacob A, et al. Treatment of neuromyelitis optica: review and recommendations. Mult Scler Relat Disord. 2012;1 (4):180-187. doi:10.1016/j.msard.2012.06.002

6. Varrin-Doyer M, Spencer CM, Schulze-Topphoff U, et al. Aquaporin 4-specific $\mathrm{T}$ cells in neuromyelitis optica exhibit a Th17 bias and recognize Clostridium ABC transporter. Ann Neurol. 2012;72 (1):53-64. doi:10.1002/ana.23651 
7. Uzawa A, Masahiro M, Kuwabara S. Cytokines and chemokines in neuromyelitis optica: pathogenetic and therapeutic implications. Brain Pathol. 2014;24(1):67-73. doi:10.1111/bpa.12097

8. Uzawa A, Mori M, Kuwabara S. Role of interleukin-6 in the pathogenesis of neuromyelitis optica. Clin Exp Neuroimmunol. 2013;4 (2):167-172. doi:10.1111/cen3.12024

9. Huda S, Whittam D, Bhojak M, Chamberlain J, Noonan C, Jacob A. Neuromyelitis optica spectrum disorders. Clin Med. 2019;19 (2):169-176. doi:10.7861/clinmedicine.19-2-169

10. Channappanavar R, Perlman S. Pathogenic human coronavirus infections: causes and consequences of cytokine storm and immunopathology. Seminars in Immunopathology. 2017;39 (5):529-539. doi:10.1007/s00281-017-0629-x

11. Cao W, Li T. COVID-19: towards understanding of pathogenesis. Cell Res. 2020;30(5):367-369. doi:10.1038/s41422-020-0327-4

12. Sormani MP. An Italian programme for COVID-19 infection in multiple sclerosis. Lancet Neurol. 2020;19(6):481-482. doi:10.1016/ S1474-4422(20)30147-2

13. Brownlee W, Bourdette D, Broadley S, Killestein J, Ciccarelli O. Treating multiple sclerosis and neuromyelitis optica spectrum disorder during the COVID-19 pandemic. Neurology. 2020;94 (22):949-952. doi:10.1212/WNL.0000000000009507

14. Elsone L, Kitley J, Luppe S, et al. Long-term efficacy, tolerability and retention rate of azathioprine in 103 aquaporin-4 antibody-positive neuromyelitis optica spectrum disorder patients: a multicentre retrospective observational study from the UK. Multiple Sclerosis J. 2014;20(11):1533-1540. doi:10.1177/1352 458514525870

15. Mealy MA, Wingerchuk DM, Palace J, Greenberg BM, Levy M. Comparison of relapse and treatment failure rates among patients with neuromyelitis optica: multicenter study of treatment efficacy. JAMA Neurol. 2014;71(3):324-330. doi:10.1001/jamaneurol.2013. 5699

16. Wingerchuk DM, Lennon VA, Lucchinetti CF, Pittock SJ, Weinshenker BG. The spectrum of neuromyelitis optica. Lancet Neurol. 2007;6(9):805-815. doi:10.1016/S1474-4422(07)70216-8

17. Sherman E, Han MH. Acute and chronic management of neuromyelitis optica spectrum disorder. Curr Treat Options Neurol. 2015;17 (11):48. doi:10.1007/s11940-015-0378-x

18. Kowarik MC, Soltys J, Bennett JL. The treatment of neuromyelitis optica. J Neuro Ophthalmol. 2014;34(1):70. doi:10.1097/WNO. 0000000000000102

19. Freedman MS, Selchen D, Arnold DL, et al. Treatment optimization in MS: canadian MS Working Group updated recommendations Canadian J Neurol Sci. 2013;40(3):307-323. doi:10.1017/S0317 167100014244

20. Bonnan M, Valentino R, Olindo S, Mehdaoui H, Smadja D, Cabre P. Plasma exchange in severe spinal attacks associated with neuromyelitis optica spectrum disorder. Multiple Sclerosis J. 2009;15 (4):487-492. doi:10.1177/1352458508100837

21. Bonnan M, Cabre P. Plasma exchange in severe attacks of neuromyelitis optica. Mult Scler Int. 2012;2012.

22. Keegan M, Pineda AA, McClelland RL, Darby CH, Rodriguez M, Weinshenker BG. Plasma exchange for severe attacks of CNS demyelination: predictors of response. Neurology. 2002;58 (1):143-146. doi:10.1212/WNL.58.1.143

23. Baharnoori M, Hohol M, Pavenski K, O'Connor P. Therapeutic Effect of Plasma Exchange (PLEX) in Neuromyelitis Optica (NMO): immediate and Long Term Response (S63. 005). $A A N$ Enterprises. 2014.

24. Kleiter I, Gahlen A, Borisow N, et al. Neuromyelitis optica: evaluation of 871 attacks and 1153 treatment courses. Ann Neurol. 2016;79 (2):206-216. doi:10.1002/ana.24554

25. Chang JC. Sepsis and septic shock: endothelial molecular pathogenesis associated with vascular microthrombotic disease. Thromb J. 2019;17(1):10. doi:10.1186/s12959-019-0198-4
26. Gyawali B, Ramakrishna K, Dhamoon AS. Sepsis: the evolution in definition, pathophysiology, and management. SAGE Open Med. 2019;7:2050312119835043. doi:10.1177/2050312119835043

27. Nguyen TC, Carcillo JA. Bench-to-bedside review: thrombocytopenia-associated multiple organ failure-a newly appreciated syndrome in the critically ill. Critical Care. 2006;10(6):235. doi:10.1186/ $\operatorname{cc5} 564$

28. Jacob S, Muppidi S, Guidon A, et al. Guidance for the management of myasthenia gravis (MG) and Lambert-Eaton myasthenic syndrome (LEMS) during the COVID-19 pandemic. J Neurol Sci. 2020;412.

29. Magraner MJ, Coret F, Casanova B. The effect of intravenous immunoglobulin on neuromyelitis optica. Neurología. 2013;28(2):65-72. doi:10.1016/j.nrleng.2012.03.014

30. Bakker J, Metz L. Devic's neuromyelitis optica treated with intravenous gamma globulin (IVIG). Canadian J Neurol Sci. 2004;31 (2):265-267. doi:10.1017/S0317167100053932

31. Okada K, Tsuji S, Tanaka K. Intermittent intravenous immunoglobulin successfully prevents relapses of neuromyelitis optica. Int Med. 2007;46(19):1671-1672. doi:10.2169/internalmedicine.46.0217

32. Wang Y, Jiang W, He Q, et al. A retrospective cohort study of methylprednisolone therapy in severe patients with COVID-19 pneumonia. Signal Transduct Targeted Ther. 2020;5(1):57. doi:10.10 38/s41392-020-0158-2

33. Cao W, Liu X, Bai T, et al. High-dose intravenous immunoglobulin as a therapeutic option for deteriorating patients with Coronavirus Disease 2019. Paper presented at: Open forum infectious diseases 2020.

34. Xie Y, Cao S, Dong H, et al. Effect of regular intravenous immunoglobulin therapy on prognosis of severe pneumonia in patients with COVID-19. J Infect. 2020;S0163-S4453(0120):30172-30179.

35. Altunrende B, Akdal G, Bajin MS, et al. Intravenous immunoglobulin treatment for recurrent optic neuritis. Arch Neuropsych. 2019;56 (1):3.

36. Borisow N, Mori M, Kuwabara S, Scheel M, Paul F. Diagnosis and treatment of NMO spectrum disorder and MOG-encephalomyelitis. Front Neurol. 2018;9:888. doi:10.3389/fneur.2018.00888

37. Huang W, Wang L, Zhang B, Zhou L, Zhang T, Quan C. Effectiveness and tolerability of immunosuppressants and monoclonal antibodies in preventive treatment of neuromyelitis optica spectrum disorders: A systematic review and network meta-analysis. Mult Scler Relat Disord. 2019;35:246-252. doi:10.1016/j.msard.2019.08.009

38. Araki M, Matsuoka T, Miyamoto K, et al. Efficacy of the anti-IL-6 receptor antibody tocilizumab in neuromyelitis optica: a pilot study. Neurology. 2014;82(15):1302-1306. doi:10.1212/WNL.0000000000 000317

39. Huang Q, Wang J, Zhou Y, et al. Low-dose mycophenolate mofetil for treatment of neuromyelitis optica spectrum disorders: a prospective multicenter study in South China. Front Immunol. 2018;9:2066. doi:10.3389/fimmu.2018.02066

40. Kim S-H, Huh S-Y, Lee SJ, Joung A, Kim HJ. A 5-year follow-up of rituximab treatment in patients with neuromyelitis optica spectrum disorder. JAMA Neurol. 2013;70(9):1110-1117. doi:10.1001/ jamaneurol.2013.3071

41. Li X, Mei S, Gong X, et al. Relationship between Azathioprine metabolites and therapeutic efficacy in Chinese patients with neuromyelitis optica spectrum disorders. BMC Neurol. 2017;17(1):130. doi:10.1186/s12883-017-0903-5

42. Xu Y, Wang Q, Ren H-T, et al. Comparison of efficacy and tolerability of azathioprine, mycophenolate mofetil, and cyclophosphamide among patients with neuromyelitis optica spectrum disorder: a prospective cohort study. J Neurol Sci. 2016;370:224-228. doi:10.1016/j.jns.2016.09.035

43. Tiede I, Fritz G, Strand S, et al. CD28-dependent Rac1 activation is the molecular target of azathioprine in primary human CD4+ T lymphocytes. J Clin Invest. 2003;111(8):1133-1145. doi:10.1172/ JCI16432 
44. Konidari A, El Matary W. Use of thiopurines in inflammatory bowel disease: safety issues. World J Gastrointest Pharmacol Ther. 2014;5 (2):63. doi:10.4292/wjgpt.v5.i2.63

45. Rademaker M, Baker C, Foley P, Sullivan J, Wang C. Advice regarding COVID-19 and use of immunomodulators, in patients with severe dermatological diseases. Australasian J Dermatol. 2020;61 (2):158-159. doi:10.1111/ajd.13295

46. Alhazzani W, Møller MH, Arabi YM, et al. Surviving Sepsis Campaign: guidelines on the management of critically ill adults with Coronavirus Disease 2019 (COVID-19). Intensive Care Med. 2020;1-34.

47. Gao F, Chai B, Gu C, et al. Effectiveness of rituximab in neuromyelitis optica: a meta-analysis. BMC Neurol. 2019;19(1):36. doi:10.1186/s12883-019-1261-2

48. Amor S, Baker D, Khoury SJ, Schmierer K, Giovanonni G. SARS-CoV -2 and Multiple Sclerosis: not All Immune Depleting DMTs are Equal or Bad. Ann Neurol. 2020;87(6):794-797. doi:10.1002/ana.25770

49. Kimby E. Tolerability and safety of rituximab (MabThera $\left.{ }^{\circledR}\right)$. Cancer Treat Rev. 2005;31(6):456-473. doi:10.1016/j.ctrv.2005.05.007

50. Juto A, Fink K, Al Nimer F, Piehl F. Interrupting rituximab treatment in relapsing-remitting multiple sclerosis; no evidence of rebound disease activity. Mult Scler Relat Disord. 2020;37:101468. doi:10.1016/j.msard.2019.101468

51. Lebrun C, Cohen M, Rosenthal-Allieri MA, et al. Only follow-up of memory B cells helps monitor rituximab administration to patients with neuromyelitis optica spectrum disorders. Neurol Ther. 2018;7 (2):373-383. doi:10.1007/s40120-018-0101-4

52. Lamba V, Sangkuhl K, Sanghavi K, Fish A, Altman RB, Klein TE. PharmGKB summary: mycophenolic acid pathway. Pharmacogenet Genomics. 2014;24(1):73. doi:10.1097/FPC.0000000000000010

53. Ritter M, Pirofski L-A. Mycophenolate mofetil: effects on cellular immune subsets, infectious complications, and antimicrobial activity. Transplant Infect Dis. 2009;11(4):290-297. doi:10.1111/j.13993062.2009.00407.x

54. Eugui E, Mirkovich A, Allison A. Lymphocyte-selective antiproliferative and immunosuppressive effects of mycophenolic acid in mice. Scand J Immunol. 1991;33(2):175-183. doi:10.1111/j.13653083.1991.tb03747.x

55. Song ATW, Abdala E, Bonazzi PR, Bacchella T, Machado MCC. Does mycophenolate mofetil increase the risk of cytomegalovirus infection in solid organ transplant recipients?: A mini-review. $\mathrm{Br}$ J Infect Dis. 2006;10(2):132-138.

56. Karnell JL, Karnell FG, Stephens GL, et al. Mycophenolic acid differentially impacts B cell function depending on the stage of differentiation. J Immunol. 2011;187(7):3603-3612. doi:10.4049/ jimmunol.1003319

57. Nosadini M, Gadian J, Lim M, Sartori S, Thomas T, Dale RC. Mycophenolate mofetil in paediatric autoimmune or immunemediated diseases of the central nervous system: clinical experience and recommendations. Dev Med Child Neurol. 2019;61(4):458-468. doi:10.1111/dmcn. 14020

58. Jacob A, Matiello M, Weinshenker BG, et al. Treatment of neuromyelitis optica with mycophenolate mofetil: retrospective analysis of 24 patients. Arch Neurol. 2009;66(9):1128-1133. doi:10.1001/ archneurol.2009.175

59. Abadja F, Atemkeng S, Alamartine E, Berthoux F, Mariat C. Impact of mycophenolic acid and tacrolimus on Th17-related immune response. Transplantation. 2011;92(4):396-403. doi:10.1097/ TP.0b013e3182247b5f

60. Antonio R, Silvia M. Immunosuppression drug-related and clinical manifestation of Coronavirus disease 2019: a therapeutical hypothesis. Am J Transplant. 2020;1.

61. Malaviya AN, Sharma A, Agarwal D, Kapoor S, Garg S, Sawhney S. Low-dose and high-dose methotrexate are two different drugs in practical terms. Int J Rheum Dis. 2010;13(4):288-293. doi:10.1111/ j.1756-185X.2010.01564.x
62. Kitley J, Elsone L, George J, et al. Methotrexate is an alternative to azathioprine in neuromyelitis optica spectrum disorders with aquaporin-4 antibodies. J Neurol Neurosurg Psychiatry. 2013;84 (8):918-921. doi:10.1136/jnnp-2012-304774

63. Ibrahim A, Ahmed M, Conway R, Carey JJ. Risk of Infection with Methotrexate Therapy in Inflammatory Diseases: A Systematic Review and Meta-Analysis. J Clin Med. 2019;8(1):15. doi:10.3390/ jcm8010015

64. Zisman D, McCune W, Tino G. Drug-induced pneumonitis: the role of methotrexate. Sarcoidosis Vasculitis Diffuse Lung Dis. 2001;18 (3):243-252.

65. Colvin O. An overview of cyclophosphamide development and clinical applications. Curr Pharm Des. 1999;5:555-560.

66. Ahlmann M, Hempel G. The effect of cyclophosphamide on the immune system: implications for clinical cancer therapy. Cancer Chemother Pharmacol. 2016;78(4):661-671. doi:10.1007/s00280016-3152-1

67. Poonsombudlert K, Kewcharoen J, Prueksapraopong C, Limpruttidham N. Post transplant cyclophosphamide based haplo-identical transplant versus umbilical cord blood transplant; a meta-analysis. Jpn J Clin Oncol. 2019;49(10):924-931. doi:10.1093/ jjco/hyz099

68. Atilla E, Ates C, Uslu A, et al. Prospective Analysis of Hemorrhagic Cystitis and BK Viremia in Allogeneic Hematopoietic Stem Cell Transplantation. Turkish j Haematol. 2019. doi:10.4274/tjh. galenos.2019.2019.0296

69. Silva T. Immunossupression murine model to study antiviral resistance emergence during Influenza A infection. 2019.

70. Frampton JE. Eculizumab: A Review in Neuromyelitis Optica Spectrum Disorder. Drugs. 2020;80(7):719-727. doi:10.1007/ s40265-020-01297-w

71. Jiang Y, Zhao G, Song N, et al. Blockade of the C5a-C5aR axis alleviates lung damage in hDPP4-transgenic mice infected with MERS-CoV. Em Microb Infect. 2018;7(1):1-12.

72. Diurno F, Numis FG, Porta G, et al. Eculizumab treatment in patients with COVID-19: preliminary results from real life ASL Napoli 2 Nord experience. Eur Rev Med Pharmacol Sci. 2020;24 (7):4040-4047.

73. Wingerchuk D, Pittock S, Berthele A, et al. Long-term safety and effectiveness of eculizumab in neuromyelitis optica spectrum disorder. Paper presented at: MULTIPLE SCLEROSIS JOURNAL 2019.

74. Araki M. Blockade of IL-6 signaling in neuromyelitis optica. Neurochem Int. 2019;130:104315. doi:10.1016/j.neuint.2018.10.012

75. Zhang C, Wu Z, Li J-W, Zhao H, Wang G-Q. The cytokine release syndrome (CRS) of severe COVID-19 and Interleukin-6 receptor (IL-6R) antagonist Tocilizumab may be the key to reduce the mortality. Int J Antimicrob Agents. 2020;55(5):105954. doi:10.1016/ j.ijantimicag.2020.105954

76. Francesco P, Perrone M.Tocilizumab in COVID-19 Pneumonia (TOCIVID-19) (TOCIVID-19). 2020https://clinicaltrials.gov/ct2/ show/NCT04317092. Accessed August 6, 2020.

77. Xu X, Han M, Li T, et al. Effective treatment of severe COVID-19 patients with tocilizumab. Proc Natl Acad Sci US A. 2020;117 (20):10970-10975. doi:10.1073/pnas.2005615117

78. Lallana JM, Clares RH, Guarnizo EC, et al. Efficacy and Safety of Tocilizumab as Second Line Therapy in Neuromyelitis Optica Unresponsive to Rituximab (P5. 262). AAN Enterprises. 2015.

79. Ayzenberg I, Faissner S, Tomaske L, Richter D, Behrendt V, Gold R. General principles and escalation options of immunotherapy in autoantibody-associated disorders of the CNS. Neurol Res Pract. 2019;1(1):32. doi:10.1186/s42466-019-0037-x

80. Yamamura T, Kleiter I, Fujihara K, et al. Trial of satralizumab in neuromyelitis optica Spectrum disorder. $N$ Eng J Med. 2019;381 (22):2114-2124. doi:10.1056/NEJMoa1901747 


\section{Publish your work in this journal}

Therapeutics and Clinical Risk Management is an international, peerreviewed journal of clinical therapeutics and risk management, focusing on concise rapid reporting of clinical studies in all therapeutic areas, outcomes, safety, and programs for the effective, safe, and sustained use of medicines. This journal is indexed on PubMed Central, CAS
EMBase, Scopus and the Elsevier Bibliographic databases. The manuscript management system is completely online and includes a very quick and fair peer-review system, which is all easy to use. Visit http://www.dovepress.com/testimonials.php to read real quotes from published authors.

Submit your manuscript here: https://www.dovepress.com/therapeutics-and-clinical-risk-management-journal 